\title{
Signatures of magnetic-field effects in nonsequential double ionization manifesting as backscattering for molecules versus forward scattering for atoms
}

\author{
G. P. Katsoulis $\odot,{ }^{1}$ M. B. Peters $\odot,{ }^{1}$ A. Staudte, ${ }^{2}$ R. Bhardwaj, ${ }^{3}$ and A. Emmanouilidou ${ }^{1}$ \\ ${ }^{1}$ Department of Physics and Astronomy, University College London, Gower Street, London WC1E 6BT, United Kingdom \\ ${ }^{2}$ Joint Attosecond Laboratory, National Research Council and University of Ottawa, Ottawa, Ontario K1A OR6, Canada \\ ${ }^{3}$ Department of Physics, University of Ottawa, Ottawa, Ontario K1N 6N5, Canada
}

(Received 14 December 2020; accepted 5 March 2021; published 22 March 2021)

\begin{abstract}
For two-electron diatomic molecules, we investigate magnetic field effects in nonsequential double ionization where recollisions prevail. We do so by formulating a three-dimensional semiclassical model that fully accounts for the Coulomb singularities and for magnetic field effects during time propagation. Using this model, we identify a prominent signature of nondipole effects. Namely, we demonstrate that the recolliding electron backscatters along the direction of light propagation. Hence, this electron escapes opposite to the direction of change in momentum due to the magnetic field. This is in striking contrast to strongly driven atoms where the recolliding electron forward scatters along the direction of light propagation. We attribute these distinct signatures to the different gate that the magnetic field creates jointly with a soft recollision in molecules compared to a hard recollision in atoms. These two different gates give rise, shortly before recollision, to different momenta and positions of the recolliding electron along the direction of light propagation. As a result, we show that the Coulomb forces from the nuclei act to backscatter the recolliding electron in molecules and forward scatter it in atoms along the direction of light propagation.
\end{abstract}

DOI: 10.1103/PhysRevA.103.033115

\section{INTRODUCTION}

Strongly driven systems constitute a computational challenge for $a b$ initio quantum-mechanical methods. As a result, most theoretical studies involve different degrees of approximations and are formulated in the dipole approximation. That is, the space dependence of the vector potential $\mathbf{A}$ is neglected. The magnetic field component of the Lorentz force $\mathbf{F}_{\mathbf{B}}=q \mathbf{v} \times \mathbf{B}$ on a particle with charge $q$ and velocity $\mathbf{v}$ is nonzero when we account for both space and time dependence of the vector potential A. Here, we account for magnetic field effects in the nonrelativistic regime. In this limit, nondipole effects are expected to arise when the amplitude of the electron motion due to $\mathbf{F}_{\mathbf{B}}$ is roughly equal to the Bohr radius, $\beta_{0} \approx E_{0}^{2} /(8 \omega c) \approx 1$ a.u. [1,2]. Hence, for smaller frequencies $\omega$ of the field, i.e., midinfrared wavelengths, nondipole effects arise at smaller field strengths $E_{0}$. Magnetic field effects give rise to striking features in multielectron ionization of systems driven by intense infrared and midinfrared laser fields [3-7].

Nondipole effects have been identified in a wide range of phenomena, mostly, in strongly driven atoms [8]. For instance, theoretical studies investigated such effects in multielectron ionization of $\mathrm{Ne}$ [9], as well as stabilization [10] and high-order harmonic generation [11-13]. In a pioneering experiment, the average final electron momentum along the direction of light propagation was measured in single ionization of atoms [3]. It is equal to the average kinetic energy of the electron divided by the speed of light $c$ [3]. In contrast, this momentum is zero in the dipole approximation. Following this experiment, quantum-mechanical studies computed the elec- tron momentum in the direction of light propagation [14-17]. They also computed how the photon momentum $I_{\mathrm{p}} / c$ is shared between electron and atomic ion; $I_{\mathrm{p}}$ is the ionization energy. This sharing was also measured in double ionization of $\mathrm{He}$ by single-photon absorption [18] and computed in strongly driven $\mathrm{H}_{2}{ }^{+}$with fixed nuclei [19].

Here, we focus on nondipole effects in nonsequential double ionization (NSDI). NSDI involves an electron (electron 1) that tunnel ionizes through the field-lowered Coulomb potential. Next, this electron accelerates in the laser field and comes back to the core to transfer energy to the initially bound electron (electron 2), leading to the escape of both electrons. Electron-electron correlation, a fundamental interaction, underlies this field-assisted recollision [20]. Numerous studies addressed NSDI, however, mostly in the dipole approximation $[21,22]$.

We previously identified nondipole gated double ionization, a prominent mechanism of nondipole effects in NSDI of strongly driven atoms $[23,24]$. The magnetic field jointly with the recollision act as a gate. This gate allows for double ionization to occur only for a subset of the initial momenta of the recolliding electron (electron 1) along the direction of light propagation, $+y$ axis here; see Fig. 1. Namely, the average initial momentum of the recolliding electron transverse to the electric field shifts along the $-y$ axis. This negative shift is opposite to the change in momentum along the $+y$ axis due to $\mathbf{F}_{\mathbf{B}}$, denoted by $\Delta p_{y, t_{0} \rightarrow t_{f}}^{B, 1}$. We predicted nondipole gated double ionization for intensities much smaller than expected from $\beta_{0} \approx 1$ a.u., for strongly driven $\mathrm{He}$ at $800 \mathrm{~nm}$. We found that this mechanism has a striking observable signature. The 


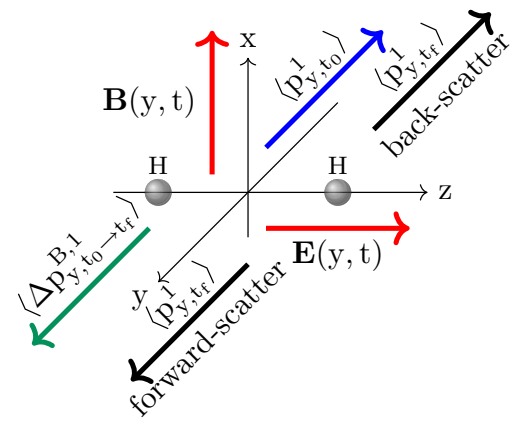

FIG. 1. Schematic illustration of the electric and magnetic field of the laser pulse as well as of the average initial and final momentum of the recolliding electron along the direction of light propagation.

recolliding electron forward scatters with a large average final momentum along the direction of light propagation. This results in the average sum of the final electron momenta being roughly an order of magnitude larger than twice the average final electron momentum in single ionization. This was verified experimentally for driven Ar at $2 \mu \mathrm{m}$ [25].

Here, we focus on the largely unexplored observable signatures of nondipole effects in nonsequential double ionization of two-electron diatomic molecules. We show that, in molecules, the recolliding electron backscatters with a large average final momentum opposite to the direction of light propagation, i.e., along the $-y$ axis. That is, the average electron momentum is opposite to the direction of the momentum gain due to $\mathbf{F}_{\mathbf{B}}$; see Fig. 1. This occurs mostly when the polarization of the electric field is along the molecular axis. Our prediction is experimentally accessible, since diatomic molecules such as $\mathrm{H}_{2}$, which is the focus of the current work, can be aligned [26]. Moreover, it takes place for intensities significantly smaller than expected from $\beta_{0} \approx 1$ a.u. As a result, the average sum of the final electron momenta is in the opposite direction and roughly an order of magnitude larger than twice the average final momentum in single ionization. Hence, nondipole effects in NSDI have strikingly different signatures in diatomic molecules versus atoms. The recolliding electron backscatters in molecules, depending on polarization, while it forward scatters in atoms [23,24].

This difference between molecules and atoms is explained in terms of "soft" [27,28] versus "hard" recollisions in nonsequential double ionization. In a hard recollision, electron 1 returns close to the core. It transfers energy to electron 2 via a long trajectory at times roughly equal to $2 T / 3 ; T$ is the period of the laser field. In a soft recollision, electron 1 does not return as close to the core. It transfers energy to electron 2 via a short trajectory at times roughly equal to $T / 2$. It is well known that in a hard recollision the recolliding electron transfers energy to a bound electron through two different pathways. In the direct one, the energy transferred suffices for both electrons to ionize shortly after recollision. In the delayed pathway, the energy transferred ionizes one of the two electrons shortly after recollision. The other electron transitions to an excited state and ionizes later. In contrast, in a soft recollision, electron 1 ionizes much earlier than the recollision time at the time electron 1 tunnel ionizes through the field-lowered Coulomb potential. Soft recollisions prevail at higher intensities than the ones where the direct pathway of a hard recollision prevails. The delayed pathway is prominent at small intensities [27,28]. For the intensities considered here, only the direct pathway of a hard recollision is of relevance. Hence, in what follows, we refer to the direct pathway of a hard recollision as hard recollision. The two electrons escape with a significant probability antiparallel to each other along the electric field for a soft recollision, while they escape parallel to each other for a hard one.

We demonstrate signatures of nondipole effects in molecules for $\mathrm{H}_{2}$ driven at $2000 \mathrm{~nm}$. We find that magnetic field effects are most prominent for intensities where soft recollisions prevail for $\mathrm{H}_{2}$, while hard ones prevail for atoms $[23,24]$. Here, we employ an $\mathrm{H}_{2}$-like model atom to allow for a direct comparison with $\mathrm{H}_{2}$. We show that the magnetic field creates a less selective gate when it acts jointly with a soft compared to a hard recollision. The main differences between these two gates are as follows. Soft recollisions compared to hard ones cause a smaller negative shift of the average initial momentum of the recolliding electron along the $-y$ axis. Moreover, shorty before a recollision, there are three main differences: (i) For a soft (compared to a hard) recollision, the initial momentum maps to much smaller values of positive momenta of electron 1; (ii) electron 1 has mostly small positive positions for a soft recollision versus mostly large negative positions for a hard one; and (iii) due to differences (i) and (ii), the Coulomb attractive forces from the nuclei backscatter electron 1 for a soft recollision while they forward scatter it for a hard one. Backscattering of the recolliding electron occurs for a wide range of wavelengths and intensities. For these parameters, $\beta_{0}$ is significantly smaller than one where magnetic fields are expected to arise.

In the current study of strongly driven $\mathrm{H}_{2}$, we keep the nuclei fixed. The reason is that NSDI prevails double ionization of $\mathrm{H}_{2}$ for fixed but not for Coulomb exploding nuclei. As a result, nondipole gated double ionization prevails double ionization mostly for strongly driven $\mathrm{H}_{2}$ with fixed nuclei. Our computations can serve as benchmark for molecules which doubly ionize via recollisions and Coulomb explode much slower than the duration of the laser pulse. Re-enforcing the applicability of our findings for fixed nuclei, we also identify signatures of electron 1 backscattering in differential observables of double ionization during the breakup of driven $\mathrm{H}_{2}$.

Finally, we formulate a three-dimensional semiclassical technique that accounts for both Coulomb singularities and nondipole effects during time propagation of driven twoelectron diatomic molecules. Employing this technique allows us to demonstrate the above-mentioned magnetic field effects in molecules. This model employs a leapfrog technique for time propagation of molecules that is more involved than the one previously employed in the dipole approximation [29]. The higher complexity is due to the derivatives of the momenta depending on the momenta and the derivatives of the positions depending on the positions of the particles in the equations of motion. Our previously developed three-dimensional semiclassical technique in the dipole approximation has resulted in ionization spectra concerning Rydberg state formation of strongly driven $\mathrm{H}_{2}$ [30] and $\mathrm{D}_{3}{ }^{+}$ [31] that are in very good agreement with experimental results [32,33]. 


\section{METHOD}

Here, we formulate a three-dimensional (3D) semiclassical model for strongly driven two-electron diatomic molecules that fully accounts for magnetic field effects during time propagation. The backbone of the current model is a 3D semiclassical model for strongly driven diatomic molecules developed in the dipole approximation [29]. Successes of this latter model include identifying the prevalent pathways of Rydberg formation during the breakup of strongly driven $\mathrm{H}_{2}$ [30] and $\mathrm{D}_{3}+$ [31]. Our results for Rydberg formation were in very good agreement with experimental results $[32,33]$. There is a major difference between the current and the previous 3D semiclassical model. Here, in the equations of motion, due to the force $\mathbf{F}_{\mathbf{B}}$, the derivatives of the positions depend on the positions and the derivatives of the momenta depend on the momenta of the particles. Hence, to integrate the equations of motion that include nondipole effects, we adopt a leapfrog technique $[34,35]$ more involved than the one used in the dipole approximation [29].

We use the initial state employed in the 3D semiclassical model in the dipole approximation. The time $t_{0}$ when the time propagation starts is selected randomly within the time interval of nonzero values of the electric field. The nuclei are initiated at rest, since we previously found that an initial predissociation does not significantly alter the ionization dynamics [29]. The initially bound electron 2 is described by a microcanonical distribution [29]. If the electric field strength at time $t_{0}$ is within the below-the-barrier ionization regime, electron 1 tunnel ionizes through the field-lowered Coulomb potential. This is also the time the propagation starts. We employ a nonrelativistic quantum-mechanical formula to compute the tunneling rate [36]. We assume that electron 1 tunnels along the direction of the electric field with zero momentum. The momentum transverse to the electric field is described by a Gaussian distribution that represents the Gaussian-shaped filter with an intensity-dependent width arising from standard tunneling theory [37-39]. In the dipole approximation, the Gaussian distribution is centered around zero. If the field strength is within the over-the-barrier ionization regime, electron 1 tunnel ionizes at the maximum of the field-lowered Coulomb potential. We set the kinetic energy of electron 1 equal to the difference between the first ionization energy of $\mathrm{H}_{2}$ and the maximum of the field-lowered Coulomb potential [29]. The polar and azimuthal angles of the momentum of electron 1 are selected randomly with the restriction that this momentum is opposite to the direction of the electric field [29]. Hence, the initial transverse electron momentum is centered around zero.

During time propagation of strongly driven $\mathrm{H}_{2}$, each electron is allowed to tunnel if the nuclei are moving, while tunneling is switched off if the nuclei are fixed. The reason we do so is that NSDI prevails double ionization for driven $\mathrm{H}_{2}$ with fixed nuclei. However, NSDI competes with enhanced ionization [40-44] during the breakup of strongly driven $\mathrm{H}_{2}$. To account for enhanced ionization, we need to allow each electron to tunnel during time propagation. Enhanced ionization occurs at a critical distance of the nuclei. A doublepotential well is formed such that it is easier for an electron bound to the higher potential well to tunnel to the lower one and then ionize. For moving nuclei, we allow for each electron to tunnel with a nonrelativistic quantum-mechanical probability given by the Wentzel-Kramers-Brillouin approximation [29]. Moreover, in our computations of the breakup of strongly driven $\mathrm{H}_{2}$, the motion of the electrons and the nuclei are treated on an equal footing. In contrast to NSDI, enhanced ionization does not require electron-electron correlation. As a result, nondipole gated double ionization does not occur in enhanced ionization.

In this work, nondipole effects are not included in tunneling that occurs in the initial state if the field strength is below the barrier. In Ref. [45], magnetic field effects were accounted for in the initial tunneling rate of an atom via a relativistic formulation. As a result, at the exit point, the momentum of electron 1 along the direction of light propagation is centered around a nonzero momentum. For the intensities considered in this work, the largest value of this momentum is $0.04 I_{p} / c$ [45]. For $\mathrm{H}_{2}$, with $I_{p}$ equal to 0.567 a.u., this momentum is equal to $1.7 \times 10^{-4}$ a.u. This value is much smaller compared to all average final electron momenta along the $y$ axis obtained in this work. Moreover, for the results presented here, for the vast majority (at least $80 \%$ ) of double ionization events, the field strength is over the barrier. For these events, there is no extra shift due to nondipole effects in the initial state [45]. Hence, we safely ignore this initial shift for all double ionization events $[23,24]$.

For molecules, we employ the Hamiltonian of the strongly driven four-body system to propagate classically the positions and the momenta of all particles. All Coulomb forces and the interaction of each electron and nucleus with the laser field are fully accounted for with no approximation. The Hamiltonian is given by

$$
H=\sum_{i=1}^{N} \frac{\left[\tilde{\mathbf{p}}_{i}-Q_{i} \mathbf{A}\left(\mathbf{r}_{i}, t\right)\right]^{2}}{2 m_{i}}+\sum_{i=1}^{N-1} \sum_{j=i+1}^{N} \frac{Q_{i} Q_{j}}{\left|\mathbf{r}_{\mathbf{i}}-\mathbf{r}_{\mathbf{j}}\right|},
$$

where $N=4$, while $Q_{i}$ is the charge, $m_{i}$ is the mass, $\mathbf{r}_{i}$ is the position vector, and $\tilde{\mathbf{p}}_{i}$ is the canonical momentum vector of particle $i$. The mechanical momentum is given by $\mathbf{p}_{i}=\tilde{\mathbf{p}}_{i}-Q_{i} \mathbf{A}\left(\mathbf{r}_{i}, t\right)$. The results presented in Sec. III concern the mechanical momentum of the electrons. The vector potential we employ is given by

$\mathbf{A}(y, t)=-\frac{E_{0}}{\omega} \exp \left[-2 \ln (2)\left(\frac{c t-y}{c \tau}\right)^{2}\right] \sin (\omega t-k y+\phi) \hat{\mathbf{z}}$,

where $k=\omega / c$ is the wave number of the electric field and $\tau$ is the full width at half maximum of the pulse duration in intensity. The carrier envelope phase (CEP) is denoted by $\phi$. This phase is important only for small-duration laser pulses. Hence, for our computations at $2000 \mathrm{~nm}, \phi$ is zero when we consider a 40 -fs duration pulse, while it is nonzero when we consider a near-single-cycle pulse of 10-fs duration in Sec. III. The electric field is taken to be along the $z$ axis and the propagation direction is along the $+y$ axis. The molecular axis is changing depending on the direction of polarization of the electric field with respect to the molecular axis. 


\section{A. Propagation technique}

In our classical formulation, we fully account for the Coulomb singularities. Hence, an electron can approach infinitely close to the nucleus during time propagation. To ensure the accurate numerical treatment of the $N$-body problem in the laser field, we perform a global regularization. This regularization was introduced in the context of the gravitational $\mathrm{N}$-body problem [46]. Here, we integrate the equations of motion using a leapfrog technique [34,35] jointly with the Bulirsch-Stoer method $[47,48]$. This ensures numerical accuracy. Also, we employ a more involved leapfrog technique than the one previously employed in our studies of strongly driven two-electron molecules in the dipole approximation [29]. We do so since here the derivatives of the positions and the momenta depend on the quantities themselves.

\section{Equations of motion}

The Hamiltonian of the $N$-body problem is defined in Eq. (1). Next, we transform to a new coordinate system that involves the relative positions $\mathbf{q}$ and the corresponding conjugate momenta $\rho$ of the particles. We define

$$
\mathbf{q}_{i j}=\mathbf{r}_{i}-\mathbf{r}_{j}
$$

and

$$
\boldsymbol{\rho}_{\mathrm{ij}}=\frac{1}{N}\left(\tilde{\mathbf{p}}_{i}-\tilde{\mathbf{p}}_{j}-\frac{m_{i}-m_{j}}{M}\langle\boldsymbol{\rho}\rangle\right),
$$

where

$$
\langle\boldsymbol{\rho}\rangle=\sum_{i=1}^{N} \tilde{\mathbf{p}}_{i} \text { and } M=\sum_{i=1}^{N} m_{i} .
$$

The inverse transformation is given by

$$
\mathbf{r}_{i}=\frac{1}{M} \sum_{j=i+1}^{N} m_{j} \mathbf{q}_{i j}-\frac{1}{M} \sum_{j=1}^{i-1} m_{j} \mathbf{q}_{j i}+\langle\mathbf{q}\rangle,
$$

and

$$
\tilde{\mathbf{p}}_{i}=\sum_{j=i+1}^{N} \boldsymbol{\rho}_{i j}-\sum_{j=1}^{i-1} \boldsymbol{\rho}_{j i}+\frac{m_{i}}{M}\langle\boldsymbol{\rho}\rangle
$$

where

$$
\langle\mathbf{q}\rangle=\frac{1}{M} \sum_{i=1}^{N} m_{i} \mathbf{r}_{i} .
$$

One can simplify Eqs. (6) and (7) by defining a fictitious particle $k$, corresponding to each pair of particles $i j$. We define the index $k$ as

$$
k(i, j)=(i-1) N-i(i+1) / 2+j,
$$

with $j>i$ and the total number of fictitious particles being $K=N(N-1) / 2$. In addition, we define the parameters $\alpha_{i k}$ and $\beta_{i k}$, as $\alpha_{i k}=1, \beta_{i k}=m_{j} / M$ and $\alpha_{j k}=-1, \beta_{j k}=$ $-m_{i} / M$ when $k=k(i j)$; otherwise, $\alpha_{i k}=\beta_{i k}=0$. Equations (6) and (7) now take the form

$$
\tilde{\mathbf{p}}_{i}=\sum_{k=1}^{K} \alpha_{i k} \boldsymbol{\rho}_{k}+\frac{m_{i}}{M}\langle\boldsymbol{\rho}\rangle,
$$

and

$$
\mathbf{r}_{i}=\sum_{k=1}^{K} \beta_{i k} \mathbf{q}_{k}+\langle\mathbf{q}\rangle .
$$

The Hamiltonian of the system can now be written in terms of the relative positions $\mathbf{q}$ and the corresponding conjugate momenta $\rho$ as

$$
\begin{aligned}
H= & \sum_{k, k^{\prime}=1}^{K} T_{k k^{\prime}} \boldsymbol{\rho}_{k} \boldsymbol{\rho}_{k^{\prime}}+\frac{\langle\boldsymbol{\rho}\rangle^{2}}{2 M}+\sum_{k=1}^{K} \frac{U_{k}}{q_{k}} \\
& +\sum_{i=1}^{N} \frac{Q_{i}^{2}}{2 m_{i}} \mathbf{A}^{2}\left(\mathbf{r}_{i}, t\right)-\sum_{i=1}^{N} \frac{Q_{i}}{m_{i}} \tilde{\mathbf{p}}_{i} \cdot \mathbf{A}\left(\mathbf{r}_{i}, t\right),
\end{aligned}
$$

with

$$
T_{k k^{\prime}}=\sum_{i=1}^{N} \frac{\alpha_{i k} \alpha_{i k^{\prime}}}{2 m_{i}} \text { and } U_{k}=Q_{i} Q_{j} .
$$

The equations of motion are expressed as follows:

$$
\begin{aligned}
\frac{d \mathbf{q}_{k}}{d t} & =2 \sum_{k^{\prime}=1}^{K} T_{k k^{\prime}} \boldsymbol{\rho}_{k^{\prime}}-\sum_{i=1}^{N} \frac{Q_{i}}{m_{i}} \alpha_{i k} \mathbf{A}\left(\mathbf{r}_{i}, t\right), \\
\frac{d\langle\mathbf{q}\rangle}{d t} & =\frac{1}{M}\langle\boldsymbol{\rho}\rangle, \\
\frac{d \boldsymbol{\rho}_{k}}{d t} & =\frac{U_{k} \mathbf{q}_{k}}{q_{k}^{3}}+\sum_{i=1}^{N} \frac{Q_{i}}{m_{i}}\left[\tilde{\mathbf{p}}_{i}-Q_{i} \mathbf{A}\left(\mathbf{r}_{i}, t\right)\right] \cdot \frac{\partial \mathbf{A}\left(\mathbf{r}_{i}, t\right)}{\partial \mathbf{q}_{\mathbf{k}}} \\
\frac{d\langle\boldsymbol{\rho}\rangle}{d t} & =\sum_{i=1}^{N} \frac{Q_{i}}{m_{i}}\left[\tilde{\mathbf{p}}_{i}-Q_{i} \mathbf{A}\left(\mathbf{r}_{i}, t\right)\right] \cdot \frac{\partial \mathbf{A}\left(\mathbf{r}_{i}, t\right)}{\partial\langle\mathbf{q}\rangle} .
\end{aligned}
$$

\section{Time-transformed leapfrog technique}

In the equations of motion (14), the derivatives of the positions depend on the positions and the derivatives of the momenta depend on the momenta of the particles. To integrate these equations, we employ a leapfrog technique [34,35] outlined in what follows. First, we perform a time transformation $t \rightarrow s$, where

$$
d s=\Omega(\mathbf{q}) d t,
$$

with $\Omega(\mathbf{q})$ an arbitrary positive function of $\mathbf{q}$. We select the function

$$
\Omega(\mathbf{q})=\sum_{k=1}^{K} \frac{1}{\left|\mathbf{q}_{k}\right|},
$$

which forces the time step to decrease when two particles undergo a close encounter and to increase when all particles are far away from each other. The equations of motion now take the following form:

$$
\begin{aligned}
\mathbf{q}^{\prime} & =\dot{\mathbf{q}}(\mathbf{q}, \boldsymbol{\rho}, t) / \Omega(\mathbf{q}) \\
\boldsymbol{\rho}^{\prime} & =\dot{\rho}(\mathbf{q}, \boldsymbol{\rho}, t) / \Omega(\mathbf{q}) \\
t^{\prime} & =1 / \Omega(\mathbf{q}),
\end{aligned}
$$

with prime denoting the derivative with respect to the new variable $s$. To perform the integration, we use the leapfrog technique described in Refs. [34,35]. This leapfrog technique 


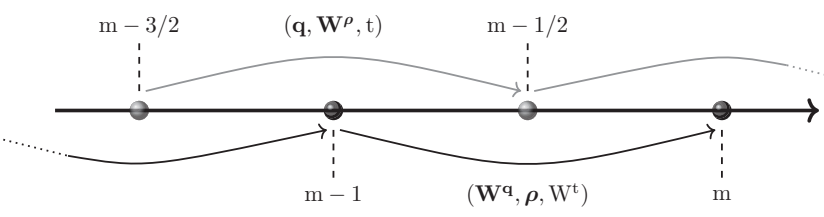

FIG. 2. Schematic illustration of the propagation of the two triplets $\left(\mathbf{q}, \mathbf{W}^{\rho}, t\right)$ and $\left(\mathbf{W}^{\mathbf{q}}, \boldsymbol{\rho}, W^{t}\right)$ over a substep of size $\mathrm{h}$, $m-3 / 2 \rightarrow m-1 / 2$ and $m-1 \rightarrow m$ respectively, with $m=$ $2, \ldots, n$.

introduces three auxiliary variables, two vectors $\mathbf{W}^{\mathbf{q}}, \mathbf{W}^{\rho}$ and one scalar $W^{t}$. As a result, an extended system is obtained where the derivatives of the position and the momenta no longer depend on the quantities themselves. The extended equations are given by

$$
\begin{aligned}
\mathbf{q}^{\prime} & =\dot{\mathbf{q}}\left(\mathbf{W}^{\mathbf{q}}, \boldsymbol{\rho}, W^{t}\right) / \Omega\left(\mathbf{W}^{\mathbf{q}}\right), \\
\mathbf{W}^{\rho^{\prime}} & =\dot{\boldsymbol{\rho}}\left(\mathbf{W}^{\mathbf{q}}, \boldsymbol{\rho}, W^{t}\right) / \Omega\left(\mathbf{W}^{\mathbf{q}}\right), \\
t^{\prime} & =1 / \Omega\left(\mathbf{W}^{\mathbf{q}}\right),
\end{aligned}
$$

and

$$
\begin{aligned}
\mathbf{W}^{\mathbf{q}^{\prime}} & =\dot{\mathbf{q}}\left(\mathbf{q}, \mathbf{W}^{\rho}, t\right) / \Omega(\mathbf{q}), \\
\rho^{\prime} & =\dot{\rho}\left(\mathbf{q}, \mathbf{W}^{\rho}, t\right) / \Omega(\mathbf{q}), \\
W^{t^{\prime}} & =1 / \Omega(\mathbf{q}) .
\end{aligned}
$$

We propagate for a time step, by propagating for half-step each triplet of variables $\left(\mathbf{q}, \mathbf{W}^{\rho}, t\right)$ and $\left(\mathbf{W}^{\mathbf{q}}, \boldsymbol{\rho}, W^{t}\right)$ in an alternating way; see the leapfrog algorithm described in the Appendix. Moreover, to achieve better accuracy, we incorporate the leapfrog method in the Bulirsch-Stoer extrapolation scheme $[47,48]$. In this scheme, a propagation over a step $H$ is split into $n$ substeps of size $h=H / n$. We use the leapfrog method to propagate over each substep. In Fig. 2, we offer a schematic illustration of the propagation during a time substep of size $h$. The detailed algorithm is described in the Appendix. This process is repeated with increasing number of substeps, i.e., $n \rightarrow \infty$, until an extrapolation with a satisfactory error is achieved.

\section{Tunneling during time propagation of $\mathrm{H}_{2}$ with moving nuclei}

During the breakup of strongly driven $\mathrm{H}_{2}$, we allow for each electron to tunnel at the classical turning points along the electric field axis using the Wentzel-Kramers-Brillouin (WKB) approximation [29]. Each electron $i$ can tunnel through a potential barrier given by

$$
\mathbf{r}_{i} \cdot \mathbf{E}\left(\mathbf{r}_{i}, t\right)+V\left(\mathbf{r}_{i}\right)
$$

where $\mathbf{E}\left(\mathbf{r}_{i}, t\right)$ is the electric field and $V\left(\mathbf{r}_{i}\right)$ is the Coulomb interaction with the nuclei. The term in the barrier involving the electric field does not appear in the Hamiltonian (1). There is no contradiction. Indeed, the gauge-invariant energy of a particle does not always coincide with the gauge-dependent Hamiltonian, as discussed in Refs. [45,49]. The energy of electron $i$ is given by

$$
\frac{\left[\tilde{\mathbf{p}}_{i}+\mathbf{A}\left(\mathbf{r}_{i}, t\right)\right]^{2}}{2}+\mathbf{r}_{i} \cdot \mathbf{E}\left(\mathbf{r}_{i}, t\right)+V\left(\mathbf{r}_{i}\right) .
$$

TABLE I. Average final electron momentum along the direction of light propagation and average kinetic energy of $\mathrm{H}_{2}$ with parallel polarization and fixed nuclei in single ionization. The Coulomb forces are either switched off or on. The values are expressed in $10^{-3}$ a.u.

\begin{tabular}{lcc}
\hline \hline & $\begin{array}{c}\text { Single ionization of } \mathrm{H}_{2} \\
\text { Coulomb off }\end{array}$ & $\begin{array}{c}\| \text { with fixed nuclei } \\
\text { Coulomb on }\end{array}$ \\
\hline$\left\langle p_{y, t_{f}}^{1}\right\rangle_{\mathrm{SI}}$ & 6 & 4 \\
$\left\langle E_{k}^{1} / c\right\rangle_{\mathrm{SI}}$ & 6 & 8 \\
\hline \hline
\end{tabular}

\section{RESULTS}

For our computations, the propagation of $\mathrm{H}_{2}$ or of the $\mathrm{H}_{2}$-like atom starts at time $t_{0}$ and ends at time $t_{f}$ when the energies of the two electrons converge. If the energies of both electrons are positive, these events correspond to double ionization (DI). Moreover, if during time propagation a recollision takes place, we label these events as NSDI. To identify a recollision, we monitor the interelectronic repulsive potential energy during time propagation. The time when this potential energy becomes maximum corresponds to the two electrons having a minimum approach. We register this time as the time of recollision, $t_{r}$. Also, we define the ionization time, $t_{i}^{\text {ion }}$, of an electron $i$ as the time when the compensated energy $\left\{p_{x, i}^{2}+p_{y, i}^{2}+\left[p_{z, i}-\hat{\mathbf{z}} \cdot \mathbf{A}\left(y_{i}, t\right)\right]^{2}\right\} / 2+V\left(\mathbf{r}_{i}\right)$ becomes positive and remains positive thereafter [50], as in previous studies [28]. For the intensities and wavelengths considered here, the vast majority of nonsequential double ionization events have only one recollision. Hence, we only consider NSDI events with one recollision. Moreover, for the pulse parameters considered here, for fixed nuclei, the majority of double ionization events are NSDI events, unless we mention otherwise. In our computations, the uncertainty of the average electron momentum is proportional to $1 / \sqrt{n_{\mathrm{ev}}}$, with $n_{\mathrm{ev}}$ being the number of events for single or double ionization. For all pulse parameters, we obtain at least $1.5 \times 10^{5}$ number of DI events, rendering the uncertainty very small.

\section{A. Strongly driven $\mathrm{H}_{2}$ with fixed nuclei}

We focus on nondipole effects in $\mathrm{H}_{2}$ with fixed nuclei driven by a laser pulse of intensity $3.5 \times 10^{14} \mathrm{~W} / \mathrm{cm}^{2}$ and duration of $40 \mathrm{fs}$ at $2000 \mathrm{~nm}$. The uncertainty in our computations of the average electron momentum is roughly $4 \times 10^{-3}$ a.u.

\section{Single ionization}

For single ionization, we compute the average of the final electron momentum $\left\langle p_{y, t_{f}}^{1}\right\rangle_{\mathrm{SI}}$, along the direction of light propagation, i.e., along the $+y$ axis; see Table I. First, we switch off all Coulomb forces. We find that the average final momentum of electron 1 is equal to its average kinetic energy, $\left\langle E_{k}\right\rangle$, divided by the speed of light $c$, as expected $[3,19]$. For Coulomb forces switched on, the average final electron momentum along the $y$ axis is still positive. However, it is equal to $4 \times 10^{-3}$ a.u. and is no longer given by $\left\langle E_{k}\right\rangle / c$, which is equal to $8 \times 10^{-3}$ a.u. 
TABLE II. Average final momentum of electron 1 and of the sum of the final electron momenta along the direction of light propagation over all double ionization events and over all NSDI events with one recollision. The values are expressed in terms of $10^{-3}$ a.u. The percentage (\%) next to NSDI denotes the fraction of NSDI events out of all double ionization events.

\begin{tabular}{|c|c|c|c|c|c|c|}
\hline \multirow[b]{2}{*}{$\alpha$} & \multicolumn{2}{|c|}{$\mathrm{H}_{2} \|$ fixed nuclei } & \multicolumn{2}{|c|}{$\mathrm{H}_{2} \perp$ fixed nuclei } & \multicolumn{2}{|c|}{$\mathrm{H}_{2}$-like atom } \\
\hline & DI & NSDI $(91 \%)$ & DI & NSDI (67\%) & DI & NSDI $(89 \%)$ \\
\hline$\left\langle p_{y, t_{0}}^{1}\right\rangle_{\alpha}$ & -18 & -14 & -8 & -11 & -36 & -35 \\
\hline$\left\langle p_{y, t_{f}}^{1}\right\rangle_{\alpha}$ & -63 & -76 & -4 & -4 & 95 & 96 \\
\hline$\left\langle p_{y, t_{f}}^{1}+p_{y, t_{f}}^{2}\right\rangle_{\alpha}$ & -61 & -78 & 6 & 3 & 137 & 140 \\
\hline
\end{tabular}

\section{Average electron momenta in double ionization}

Next, we focus on nonsequential double ionization of $\mathrm{H}_{2}$ with fixed nuclei driven by a pulse of either parallel or perpendicular polarization with respect to the molecular axis. In Table II, we compare the results for $\mathrm{H}_{2}$ with the results for the $\mathrm{H}_{2}$-like atom for NSDI and for all double ionization events. The $\mathrm{H}_{2}$-like atom is driven by the same pulse and shares the same total nuclear charge, two, and the same first and second ionization potentials as $\mathrm{H}_{2}$. For all three cases, in nonsequential double ionization, the average of the initial momentum of electron 1 along the direction of light propagation has a negative shift. That is, $\left\langle p_{y, t_{0}}^{1}\right\rangle_{\mathrm{NSDI}}$ is shifted opposite to the direction of the momentum change due to the magnetic force $\mathbf{F}_{\mathbf{B}}$. However, this initial negative shift is much larger for the $\mathrm{H}_{2}$-like atom compared to $\mathrm{H}_{2}$. Indeed, this shift for the $\mathrm{H}_{2}$-like atom has more than double (three times) the value for $\mathrm{H}_{2}$ with parallel (perpendicular) polarization. For simplicity, we refer to the momentum and position of an electron along the direction of light propagation as transverse. Moreover, for $\mathrm{H}_{2}$ with parallel polarization, the average final transverse momentum of electron 1, i.e., $\left\langle p_{y, t_{f}}^{1}\right\rangle_{\mathrm{NSDI}}$, and the average of the sum of the final transverse electron momenta, i.e., $\left\langle p_{y, t_{f}}^{1}+p_{y, t_{f}}^{2}\right\rangle_{\mathrm{NSDI}}$, are almost an order of magnitude larger than twice the average final electron momentum for single ionization, i.e., $2\left\langle p_{y, t_{f}}^{1}\right\rangle_{\mathrm{SI}}$. Similarly, we find that $\left\langle p_{y, t_{f}}^{1}\right\rangle_{\mathrm{NSDI}}$ is more than a magnitude larger than $2\left\langle p_{y, t_{f}}^{1}\right\rangle_{\mathrm{SI}}$ for the $\mathrm{H}_{2}$-like atom. However, there is a striking difference between $\mathrm{H}_{2}$ with parallel polarization and the $\mathrm{H}_{2}$-like atom. In the former case, $\left\langle p_{y, t_{f}}^{1}\right\rangle_{\mathrm{NSDI}}$ and $\left\langle p_{y, t_{f}}^{1}+p_{y, t_{f}}^{2}\right\rangle_{\mathrm{NSDI}}$ have large negative values, while in the latter case they have large positive values. Hence, on average, electron 1 backscatters for $\mathrm{H}_{2}$ with parallel polarization and forward scatters for the $\mathrm{H}_{2}$-like atom along the direction of light propagation. Forward scattering of electron 1 is in accordance with our previous finding for strongly driven $\mathrm{He}$ at $800 \mathrm{~nm}[23,24]$. Also, we find that for $\mathrm{H}_{2}$ with perpendicular polarization the average final electron momentum has small values.

We also compute NSDI for $\mathrm{H}_{2}$ with fixed nuclei when driven by a near-single-cycle laser pulse of parallel polarization, intensity of $3.5 \times 10^{14} \mathrm{~W} / \mathrm{cm}^{2}$, and duration of $10 \mathrm{fs}$ at $2000 \mathrm{~nm}$. We consider five values of $\phi$ in Eq. (2), i.e., of CEP, from $0^{\circ}$ to $360^{\circ}$ in steps of $60^{\circ}$. Such CEP-controlled near-single-cycle laser pulses have been employed in experiments of NSDI [51]. Averaging over all CEPs, we find that $\left\langle p_{y, t_{f}}^{1}+p_{y, t_{f}}^{2}\right\rangle_{\mathrm{NSDI}}=-110 \times 10^{-3}$ a.u. That is, when $\mathrm{H}_{2}$ is driven by a 10 -fs duration pulse we find that the recolliding electron backscatters, as is the case for a 40 -fs duration pulse; see Table II. This corroborates our previous statement that our results for $\mathrm{H}_{2}$ with fixed nuclei driven by a 40-fs duration pulse apply to molecules Coulomb exploding in a timescale larger than the duration of the pulse. Computations with a 40-fs duration pulse, which has no CEP dependence, are significantly less demanding than those with a 10-fs duration pulse. Hence, in what follows, we present results computed for $\mathrm{H}_{2}$ with fixed nuclei driven by a 40-fs duration pulse.

\section{Soft versus hard recollision in NSDI}

The main difference among the three cases is the relative contribution of the recollision and the laser field in the ionization of electrons 1 and 2 . For the $\mathrm{H}_{2}$-like atom, we find that the two electrons escape following a recollision which is assisted by the laser field, i.e., a hard recollision. Specifically, electron 1 returns to the core very close to the initially bound electron. It transfers energy to electron 2 at times roughly equal to $2 T / 3$ past the time $t_{0}$. Moreover, we find that the two electrons ionize at times close to the recollision time. As a result, their final momenta are roughly equal to the value of the vector potential at the time of ionization, i.e., $-\mathbf{A}\left(y_{i}, t_{i}^{\text {ion }}\right)$. Hence, both electrons escape with roughly parallel momenta along the direction of polarization; see Fig. 3(a).

For $\mathrm{H}_{2}$, we find that the two electrons escape via field ionization assisted by recollision, i.e., soft recollision, mostly so for $\mathrm{H}_{2}$ with perpendicular polarization. Specifically, we find that electron 1 does not return as close to the core as for a hard recollision. Electron 1 transfers energy to electron 2 at times roughly equal to $T / 2$ past the time $t_{0}$. Moreover, we find that electron 1 mostly ionizes shortly after time $t_{0}$. We note that electron 1 tunnel-ionizing in the initial state does not imply ionization at time $t_{0}$. Indeed, the latter occurs only if the energy of an electron satisfies the above-mentioned definition

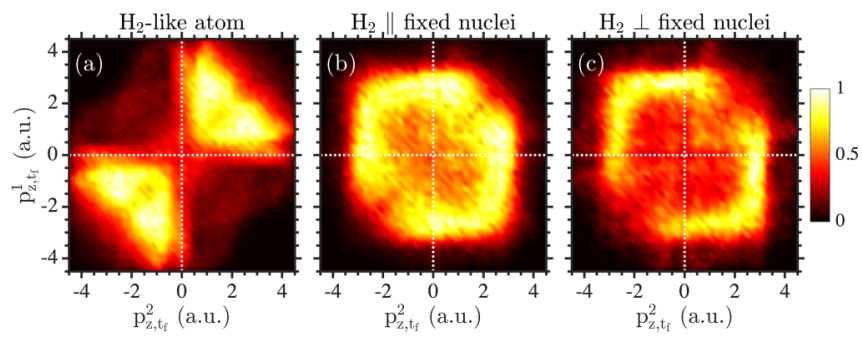

FIG. 3. Symmetrized correlated electron momenta for NSDI normalized to peak value for (a) the $\mathrm{H}_{2}$-like atom, (b) $\mathrm{H}_{2}$ with parallel polarization and fixed nuclei, and (c) $\mathrm{H}_{2}$ with perpendicular polarization and fixed nuclei. 
of ionization in terms of the compensated energy. Electron 2 ionizes at times close to the recollision time. The values of the vector potential at times that differ by half a period have opposite signs. Hence, the electrons escape with opposite momenta along the direction of polarization. This is mostly the case for $\mathrm{H}_{2}$ with perpendicular polarization; see Fig. 3(c). A more detailed description of soft recollisions is offered in Ref. [27]. For $\mathrm{H}_{2}$ with parallel polarization, the recollision is not as strong as for the $\mathrm{H}_{2}$-like atom but not as soft as for $\mathrm{H}_{2}$ with perpendicular polarization; see Fig. 3(b). This is also corroborated by NSDI prevailing in double ionization for $\mathrm{H}_{2}$ with parallel polarization (91\%) and the $\mathrm{H}_{2}$-like atom (89\%); see Table II. However, for $\mathrm{H}_{2}$ with perpendicular polarization, for a significant ratio of double ionization events the two electrons escape via field ionization. The differences between soft and hard recollisions described above are in accord with our previous findings in the dipole approximation for driven $\mathrm{N}_{2}$ with fixed nuclei and $\operatorname{Ar}[27,28]$.

The above differences between soft and hard recollisions result in the magnetic field jointly with a soft recollision creating a less restrictive gate compared to the one for a hard recollision. This is in accordance with our finding, shown in Table II , that the negative shift in the initial transverse momentum of electron 1 is much smaller for $\mathrm{H}_{2}$ compared to the $\mathrm{H}_{2}$-like atom.

\section{Electron momentum and position along the direction of light propagation shortly before recollision}

Moreover, we show that these different gates give rise to three key differences in the dynamics of electron 1 in strongly driven molecules compared to atoms along the direction of light propagation. Namely, shortly before recollision, electron 1 has mostly small positive momenta for molecules compared to large positive momenta for atoms. Also, shortly before recollision, electron 1 has mostly small positive positions for molecules compared to large negative positions for atoms. Finally, as a result of these two differences, in the time interval from shortly before recollision up to the recollision time, the Coulomb forces from the nuclei act to backscatter electron 1 for molecules and to forward scatter it for atoms.

In what follows, we demonstrate in detail these differences between $\mathrm{H}_{2}$ and the $\mathrm{H}_{2}$-like atoms, as well as between the parallel and perpendicular polarizations of $\mathrm{H}_{2}$. In all three cases, the initial transverse momentum of electron 1 is shifted toward negative values at time $t_{0}$; see Fig. 4(a1). Shortly before recollision, we find that the transverse momentum of electron 1 has mostly large positive values for the $\mathrm{H}_{2}$-like atom and mostly small positive values for $\mathrm{H}_{2}$; see Fig. 4(a2). Moreover, for $\mathrm{H}_{2}$, the transverse momentum of electron 1 has smaller positive values for the parallel compared to the perpendicular polarization; see Fig. 4(a2). For $\mathrm{H}_{2}$, it is these small positive values of the transverse electron momentum that give rise to negative values of the average final momentum of electron 1 along the $y$ axis. This is clearly shown in Figs. 5(a) and 5(b). Namely, the electron momenta in the interval [0, $0.02]$ a.u. shortly before recollision contribute the most to the average final momentum of electron 1 being negative for $\mathrm{H}_{2}$. In contrast, for the $\mathrm{H}_{2}$-like atom, all electron momenta shortly before recollision contribute to positive values of the average

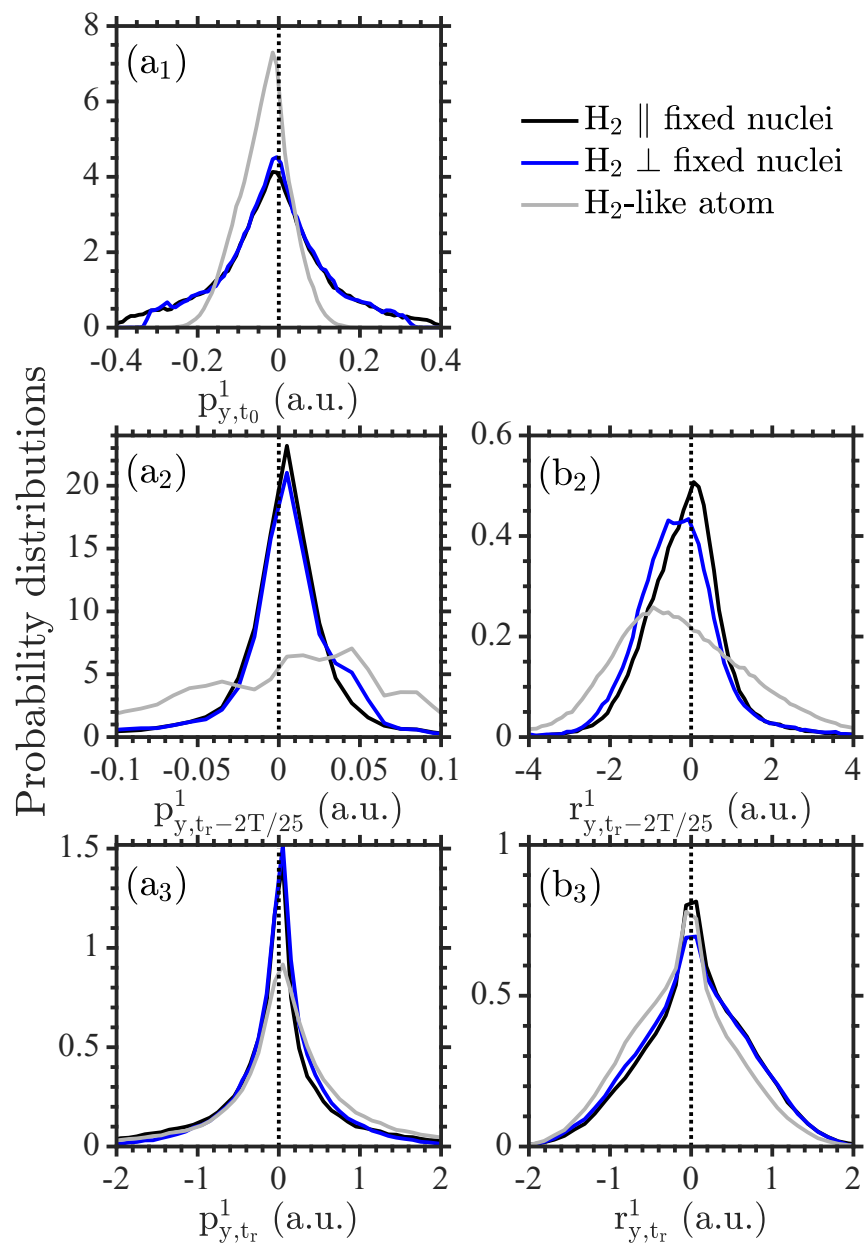

FIG. 4. Plots of the distribution of the transverse momentum (a) and position (b) of electron 1 at the time of tunnel ionization $t_{0}$ (a1), shortly before recollision at time $t_{r}-2 T / 25$ [(a2),(b2)], and at the time of recollision $t_{r}[(\mathrm{a} 3),(\mathrm{b} 3)]$.

final electron momentum along the $y$ axis; see Fig. 5(c). Also, comparison of the color scale in Fig. 5(a) with that in Fig. 5(b) shows that the small electron momenta contribute larger negative values to the average final electron momentum for the parallel compared to the perpendicular polarizations.

Next, shortly before recollision, we investigate the distribution of the position of electron 1 along the direction of light propagation. In all three cases, the position of electron 1 along the $y$ axis is zero at the exit point at time $t_{0}$. We find that electron 1 has significantly larger negative values for the $\mathrm{H}_{2}$-like atom compared to $\mathrm{H}_{2}$; see Fig. 4(b2). This difference is in accordance with the significantly larger negative shift of the initial transverse momentum of electron 1 for the $\mathrm{H}_{2}$-like atom. Moreover, for $\mathrm{H}_{2}$, Fig. 4(b2) shows that the distribution of the position along the $y$ axis of electron 1 peaks at small positive values for parallel polarization. However, it peaks at small negative values for perpendicular polarization.

\section{Effect of the Coulomb forces from the nuclei in the time interval from shortly before recollision up to recollision}

Shortly before recollision, the distributions of the momentum and the position of electron 1 along the direction of light 

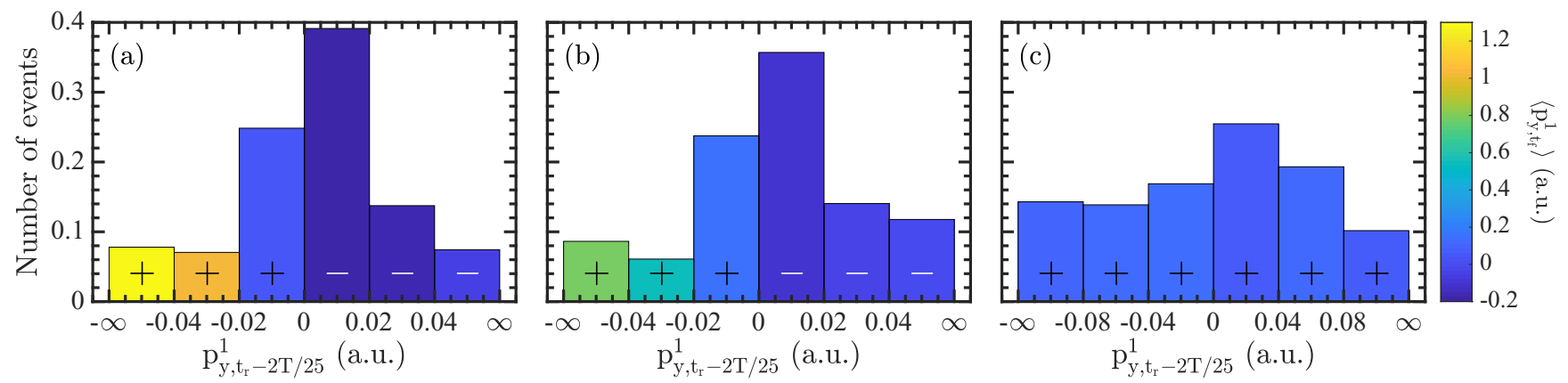

FIG. 5. Average final momentum of electron 1 along the $y$ axis, given by the color scale, as a function of the momentum of electron 1 along the $y$ axis shortly before recollision at time $t_{r}-2 T / 25$ (a) for $\mathrm{H}_{2}$ with parallel polarization and fixed nuclei, (b) for $\mathrm{H}_{2}$ with perpendicular polarization and fixed nuclei, and (c) for the $\mathrm{H}_{2}$-like atom. The + and - signs are used to facilitate the correct interpretation of the color scale. The heights of the columns denote the relative contribution of NSDI events with momenta at time $t_{r}-2 T / 25$ in a certain momentum interval to the total number of NSDI events.

propagation differ between molecule and atom as well as between parallel and perpendicular polarization of $\mathrm{H}_{2}$. This results in the Coulomb attractive forces exerted on electron 1 from the nuclei having a different effect on the dynamics of electron 1. This effect is more pronounced in the time interval from shortly before recollision up to the time of recollision.

Indeed, we quantify the contribution of the Coulomb forces as follows. The average value of the final electron momentum along the direction of light propagation is equal to

$$
\begin{aligned}
\left\langle p_{y}^{1}, t_{f}\right\rangle= & \left\langle p_{y}^{1}, t_{0}\right\rangle+\left\langle\Delta p_{y, t_{0} \rightarrow t_{f}}^{C, 1}\right\rangle+\left\langle\Delta p_{y, t_{0} \rightarrow t_{f}}^{B, 1}\right\rangle \\
= & \left\langle p_{y}^{1}, t_{0}\right\rangle+\left\langle\Delta p_{y, t_{0} \rightarrow t_{b}}^{C, 1}\right\rangle+\left\langle\Delta p_{y, t_{b} \rightarrow t_{r}}^{C, 1}\right\rangle \\
& +\left\langle\Delta p_{y, t_{r} \rightarrow t_{a}}^{C, 1}\right\rangle+\left\langle\Delta p_{y, t_{a} \rightarrow t_{f}}^{C, 1}\right\rangle+\left\langle\Delta p_{t_{0} \rightarrow t_{f}}^{B, 1}\right\rangle,
\end{aligned}
$$

with

$$
\begin{aligned}
& \Delta p_{y, t_{1} \rightarrow t_{2}}^{C, 1}=\int_{t_{1}}^{t_{2}}\left(\frac{R_{y}^{1}-r_{y}^{1}}{\left|\mathbf{R}_{1}-\mathbf{r}_{1}\right|^{3}}+\frac{R_{y}^{2}-r_{y}^{1}}{\left|\mathbf{R}_{2}-\mathbf{r}_{1}\right|^{3}}+\frac{r_{y}^{1}-r_{y}^{2}}{\left|\mathbf{r}_{2}-\mathbf{r}_{1}\right|^{3}}\right) d t \\
& \Delta p_{y, t_{1} \rightarrow t_{2}}^{B, 1}=\int_{t_{1}}^{t_{2}} F_{B} d t
\end{aligned}
$$

where $\Delta p_{y, t_{1} \rightarrow t_{2}}^{C / B, 1}$ denotes the change in momentum of electron 1 in the time interval $\left[t_{1}, t_{2}\right]$ due to the Coulomb forces $(C)$ and the magnetic field $(B)$. The times $t_{b}$ and $t_{a}$ correspond to times shortly before $t_{r}-T / 10$ and shortly after $t_{r}+T / 10$ recollision. The electric field is always transverse to the direction of light propagation. Hence, it does not contribute to the change in the electron momentum along the $y$ axis. The Coulomb forces include the attractive forces from the nuclei and the repulsive one from electron 2 . However, the largest contribution arises from the Coulomb forces from the nuclei. The change in electron momentum due to the magnetic field is computed from the start of the propagation $t_{0}$ until the final time $t_{f}$, i.e., $\left\langle\Delta p_{t_{0} \rightarrow t_{f}}^{B, 1}\right\rangle$. The reason is that this change in electron momentum is roughly the same in all three cases; see Fig. 6(b). Also, it is much smaller compared to the change in momentum due to the Coulomb forces for $\mathrm{H}_{2}$ with parallel polarization and the $\mathrm{H}_{2}$-like atom; see Fig. 6(b). In Fig. 6(a), it is clearly shown that the change in momentum of electron 1 due to the Coulomb forces arises mainly in the time interval from shortly before recollision, $t_{r}-T / 10$, up to the recollision time, $t_{r}$.

For the $\mathrm{H}_{2}$-like atom, Fig. 6(a) shows that the change in momentum of electron 1 along the $y$ axis due to the Coulomb forces is large and positive in the time interval from shortly before recollision and up to the recollision time. This is consistent with our finding in Sec. III A 4 that, shortly before recollision, electron 1 has mostly large negative values of the position and mostly large positive momenta along the $y$ axis. Hence, the Coulomb forces from the nuclei act to mostly accelerate electron 1 along the $+y$ axis in this time interval.

In contrast, for $\mathrm{H}_{2}$ with parallel polarization, Fig. 6(a) shows that the change in momentum of electron 1 along the $y$ axis due to the Coulomb forces is large and negative in the time interval from shortly before recollision and up to the recollision time. This is in accordance with our finding in Sec. III A 4 that electron 1 has mostly small positive values of the position and mostly small positive momenta along the $y$ axis. As a result, the Coulomb attractive forces from the nuclei decelerate electron 1 and easily overtake the initially small positive momenta. This leads to electron 1 eventually accelerating along the $-y$ axis and, thus, backscattering. For $\mathrm{H}_{2}$ with perpendicular polarization, electron 1 still has mostly small positive momenta shortly before recollision. However, it has mostly small negative positions along the $y$ axis. As a result, the Coulomb forces act first to accelerate electron 1. Hence, electron 1 crosses over to the $+y$ axis with larger positive momenta than for the case of parallel polarization. As a result, the Coulomb forces accelerate electron 1 for a smaller time along the $-y$ axis. This results in the change in electron momentum due to the Coulomb forces being much smaller for $\mathrm{H}_{2}$ with perpendicular compared to parallel polarization; see Fig. 6(a).

\section{B. Backscattering of electron 1 dependence on frequency and intensity for strongly driven $\mathrm{H}_{2}$ with fixed nuclei}

Next, we demonstrate that backscattering of electron 1 is a prominent nondipole effect for a wide range of wavelengths and intensities for two-electron diatomic molecules. Indeed, in Fig. 7(a), for $\mathrm{H}_{2}$ with parallel polarization and fixed nuclei, we show the dependence on wavelength of the 


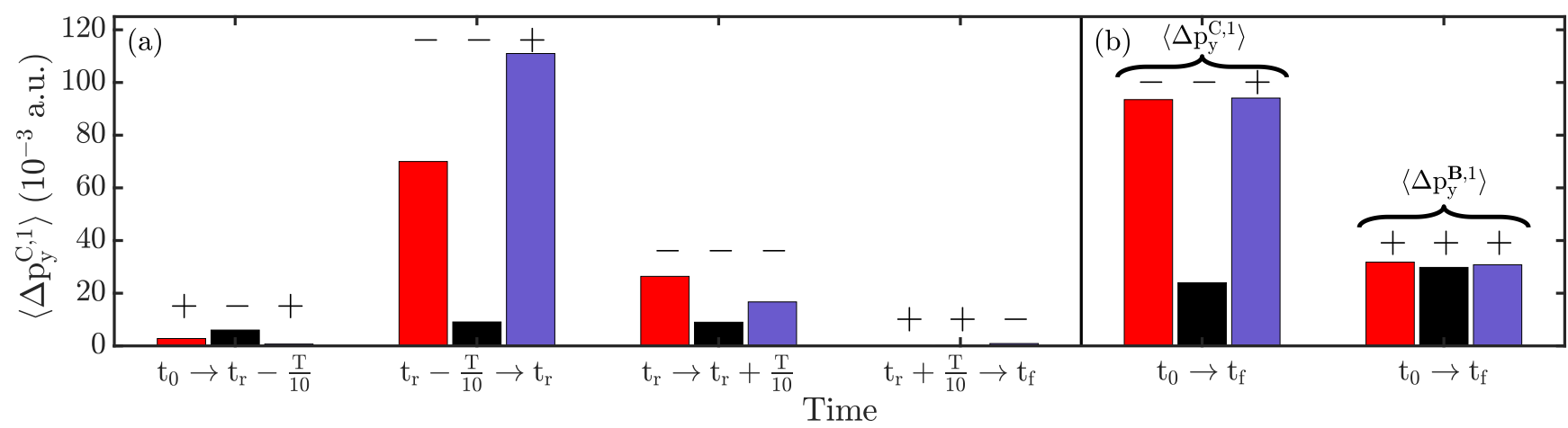

FIG. 6. Change in momentum of electron 1 due to (a) the Coulomb forces in different time intervals and (b) the total contribution to the change in momentum of electron 1 due to the Coulomb forces and due to the magnetic field. In both panels (a) and (b), the three columns in each time interval correspond, from left to right, to $\mathrm{H}_{2}$ with parallel polarization and fixed nuclei (red), to $\mathrm{H}_{2}$ with perpendicular polarization and fixed nuclei (black), and to the $\mathrm{H}_{2}$-like atom (purple).

average initial electron momentum, the average final electron momentum and the average of the sum of the final electron momenta. Keeping the intensity fixed at $3.5 \times 10^{14} \mathrm{~W} / \mathrm{cm}^{2}$, we find that the negative values of all three average electron momenta persist for wavelengths that range from 2000 down to $800 \mathrm{~nm}$. However, the negative values of these average electron momenta are large and close to the their values at $2000 \mathrm{~nm}$ mainly for wavelengths down to $1400 \mathrm{~nm}$. The negative values of these average momenta increase gradually. This is consistent with the change in the distribution of the momentum [Fig. 7(b)] and position [Fig. 7(c)] of electron 1 along the propagation direction shortly before recollision, at time $t_{r}-2 T / 25$. Indeed, as the wavelength decreases from 2000 to $800 \mathrm{~nm}$, we find that electron 1 shortly before recollision has gradually increasing momenta and increasing positive positions along the $y$ axis. These changes result in electron 1 forward scattering for more NSDI events for wavelengths smaller than $2000 \mathrm{~nm}$.

Also, in Fig. 8(a), for $\mathrm{H}_{2}$ driven at $2000 \mathrm{~nm}$ with parallel polarization and fixed nuclei, we show the dependence on intensity of the average initial electron momentum, the average final electron momentum, and the average of the sum of the final electron momenta. We find that the negative values of all three average electron momenta persist for intensities from $3.5 \times 10^{14} \mathrm{~W} / \mathrm{cm}^{2}$ down to $2.25 \times 10^{14} \mathrm{~W} / \mathrm{cm}^{2}$. The negative values of these average momenta increase gradually.
This is consistent with the change in the distribution of the momentum [Fig. 8(b)] and position [Fig. 8(c)] of electron 1 along the propagation direction shortly before recollision, at time $t_{r}-2 T / 25$. As the intensity decreases, we find that electron 1 shortly before recollision has gradually increasing momenta and increasing positive positions along the $y$ axis. These changes result in electron 1 scattering forwards for more NSDI events for smaller intensities. This dependence on intensity suggests that backscattering of electron 1 will be observable even if volume averaging, i.e., integration over different intensities, is included.

\section{Strongly driven $\mathrm{H}_{2}$ with moving nuclei}

Next, we show that backscattering of electron 1 along the propagation direction is also present during the Coulomb explosion of the nuclei of strongly driven $\mathrm{H}_{2}$ with parallel polarization. As discussed above, during the breakup of twoelectron diatomic molecules nonsequential double ionization competes with enhanced ionization. Nondipole gated double ionization is not present in enhanced ionization. Hence, in order to increase the contribution of NSDI compared to enhanced ionization, we consider a short-duration laser pulse. That is, $\mathrm{H}_{2}$ is driven by a near-single-cycle laser field of intensity $3.5 \times 10^{14} \mathrm{~W} / \mathrm{cm}^{2}$ and $10 \mathrm{fs}$ duration at $2000 \mathrm{~nm}$. We consider five values of $\phi$ in Eq. (1), i.e., of CEP, from $0^{\circ}$ to
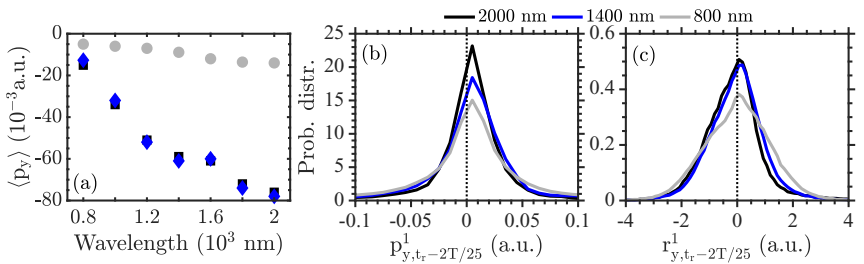

FIG. 7. In NSDI, for $\mathrm{H}_{2}$ with parallel polarization with fixed nuclei driven by a laser pulse of intensity $3.5 \times 10^{14} \mathrm{~W} / \mathrm{cm}^{2}$, average initial transverse momentum of electron 1 (gray circles), average final transverse momentum of electron 1 (black squares), and average of the sum of the final electron momenta (blue diamonds), as a function of wavelength (a). Plots of the distribution of the transverse momentum (b) and transverse position (c) of electron 1 shortly before recollision at time $t_{r}-2 T / 25$ for three wavelengths.
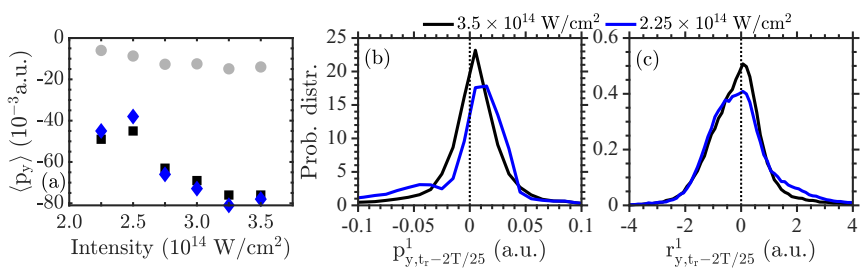

FIG. 8. In NSDI, for $\mathrm{H}_{2}$ with parallel polarization with fixed nuclei driven at $2000 \mathrm{~nm}$, average initial transverse momentum of electron 1 (gray circles), average final transverse momentum of electron 1 (black squares), and average of the sum of the final electron momenta (blue diamonds), as a function of intensity (a). Plots of the distribution of the transverse momentum (b) and transverse position (c) of electron 1 shortly before recollision at time $t_{r}-2 T / 25$ for three intensities. 

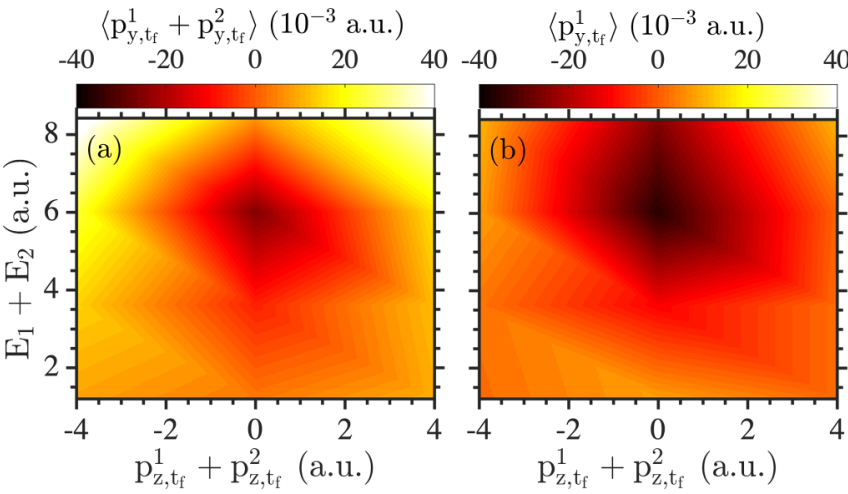

FIG. 9. Average of the sum of the final electron momenta along the propagation direction (color scale) (a) and average of the final momentum of electron 1 (color scale) (b) as a function of the sum of the final electron energies and of the sum of the final electron momenta along the direction of light polarization.

$360^{\circ}$ in steps of $60^{\circ}$. The results presented below are obtained by averaging over the five values of $\phi$.

For these laser pulse parameters, we find that enhanced ionization events are more than half of all double ionization events. Hence, over all double ionization events, it is unlikely that negative values of the average sum of the final electron momenta will be observed experimentally. However, we show that such negative values can be observed when considering doubly differential probabilities. Indeed, as discussed in Sec. III A 3, a signature of soft recollisions is that the two electrons escape opposite to each other along the direction of polarization; see Figs. 3(b) and 3(c). For these events, the two electrons escape with antiparallel but large momenta. Hence, for the events that favor electron 1 backscattering, $p_{z, t_{f}}^{1}+p_{z, t_{f}}^{2} \approx 0$ and the sum of the final electron energies $\mathrm{E}_{1}+\mathrm{E}_{2}$ will have values much larger than zero. Indeed, in Fig. 9(a), we show that when both $p_{z, t_{f}}^{1}+p_{z, t_{f}}^{2}$ is close to zero and $E_{1}+E_{2}$ is roughly equal to 6 a.u., the average sum of the final electron momenta along the $y$ axis is roughly equal to $-25 \times 10^{-3}$ a.u. This negative value is due to electron 1 backscattering. Indeed, for the same conditions, Fig. 9(b) shows that electron 1 has a negative average final electron momentum roughly equal to $-35 \times 10^{-3}$ a.u.

\section{CONCLUSIONS}

We identify a striking signature of nondipole effects in nonsequential double ionization of strongly driven two-electron diatomic molecules. Namely, the average final momentum of the recolliding electron along the direction of light propagation has a large negative value. As a result, the average sum of the final electron momenta along the direction of light propagation is also large and negative. This large negative values are in contrast to the large positive values for the same quantities in double ionization of strongly driven atoms. Hence, the recolliding electron backscatters for strongly driven two-electron diatomic molecules while it forward scatters for strongly driven atoms. We find this to be the case for intensities much smaller than expected for magnetic fields to arise. Also, we find that backscattering of the recolliding electron persists for a wide range of frequencies and intensities.

The intensities where both magnetic fields effects and recollisions are present correspond to soft recollisions for $\mathrm{H}_{2}$ and hard recollisions for two-electron atoms. Back- versus forward scattering of the recolliding electron stems from the magnetic field creating a different gate jointly with a soft recollision for molecules compared to a hard recollision for atoms. Both gates result in the initial momentum of the recolliding electron being mostly negative along the direction of light propagation. However, this negative shift is smaller for a soft recollision compared to a hard one. These different gates result in three main differences shortly before recollision between molecules and atoms along the direction of light propagation. The recolliding electron has mostly much smaller positive momenta for molecules compared to atoms. Also, it has mostly small positive positions for molecules versus large negative positions for atoms. As a result of the above two differences, the Coulomb forces from the nuclei act to backscatter the recolliding electron in two-electron molecules versus forward scatter it in atoms.

Backscattering of the recolliding electron gives rise to large negative values of the average sum of the final electron momenta in driven two-electron diatomic molecules. This was demonstrated in the context of $\mathrm{H}_{2}$ with fixed nuclei driven by a pulse with parallel polarization at $2000 \mathrm{~nm}$. Our computations serve as benchmark for molecules which doubly ionize via recollisions and Coulomb explode much slower than the duration of the laser pulse. To re-enforce the applicability of our computations for molecules with fixed nuclei, we also consider the breakup of $\mathrm{H}_{2}$ driven by near-single-cycle laser pulses at $2000 \mathrm{~nm}$. We show that signatures of backscattering of the recollinding electron are still found when considering doubly differential probabilities of double ionization.

\section{ACKNOWLEDGMENTS}

A.E. acknowledges the EPSRC Grant EP/N031326/1 and the use of the UCL Myriad High Throughput Computing Facility (Myriad@UCL), and associated support services, in the completion of this work.

\section{APPENDIX: LEAPFROG ALGORITHM}

In what follows, we describe the leapfrog algorithm. First, we initialize the auxiliary variables, $\mathbf{W}_{\mathbf{0}}^{\mathbf{q}}=\mathbf{q}_{0}, \mathbf{W}_{\mathbf{0}}^{\rho}=$ $\rho_{0}, W_{0}^{t}=t_{0}$. Then, we propagate for a time step equal to $h$, by propagating for half a step each triplet of variables $\left(\mathbf{q}, \mathbf{W}^{\rho}, t\right)$ and $\left(\mathbf{W}^{\mathbf{q}}, \boldsymbol{\rho}, W^{t}\right)$ in an alternating way as follows:

$$
\begin{aligned}
\mathbf{q}_{1 / 2} & =\mathbf{q}_{0}+\frac{h}{2} \frac{\dot{\mathbf{q}}\left(\mathbf{W}_{0}^{\mathbf{q}}, \boldsymbol{\rho}_{0}, W_{0}^{t}\right)}{\Omega\left(\mathbf{W}_{0}^{\mathbf{q}}\right)}, \\
\mathbf{W}_{1 / 2}^{\rho} & =\mathbf{W}_{0}^{\rho}+\frac{h}{2} \frac{\dot{\rho}\left(\mathbf{W}_{0}^{\mathbf{q}}, \boldsymbol{\rho}_{0}, W_{0}^{t}\right)}{\Omega\left(\mathbf{W}_{0}^{\mathbf{q}}\right)}, \\
t_{1 / 2} & =t_{0}+\frac{h}{2} \frac{1}{\Omega\left(\mathbf{W}_{0}^{\mathbf{q}}\right)}, \\
\mathbf{W}_{1}^{\mathbf{q}} & =\mathbf{W}_{0}^{\mathbf{q}}+h \frac{\dot{\mathbf{q}}\left(\mathbf{q}_{1 / 2}, \mathbf{W}_{1 / 2}^{\rho}, t_{1 / 2}\right)}{\Omega\left(\mathbf{q}_{1 / 2}\right)},
\end{aligned}
$$




$$
\begin{aligned}
\rho_{1} & =\rho_{0}+h \frac{\dot{\rho}\left(\mathbf{q}_{1 / 2}, \mathbf{W}_{1 / 2}^{\rho}, t_{1 / 2}\right)}{\Omega\left(\mathbf{q}_{1 / 2}\right)}, \\
W_{1}^{t} & =W_{0}^{t}+h \frac{1}{\Omega\left(\mathbf{q}_{1 / 2}\right)}, \\
\mathbf{q}_{1} & =\mathbf{q}_{1 / 2}+\frac{h}{2} \frac{\dot{\mathbf{q}}\left(\mathbf{W}_{1}^{\mathbf{q}}, \rho_{1}, W_{1}^{t}\right)}{\Omega\left(\mathbf{W}_{1}^{\mathbf{q}}\right)} \\
\mathbf{W}_{1}^{\rho} & =\mathbf{W}_{1 / 2}^{\rho}+\frac{h}{2} \frac{\dot{\rho}\left(\mathbf{W}_{1}^{\mathbf{q}}, \rho_{1}, W_{1}^{t}\right)}{\Omega\left(\mathbf{W}_{1}^{\mathbf{q}}\right)}, \\
t_{1} & =t_{1 / 2}+\frac{h}{2} \frac{1}{\Omega\left(\mathbf{W}_{1}^{\mathbf{q}}\right)} .
\end{aligned}
$$

The subscripts $0,1 / 2$, and 1 denote the values of each variable at the initial, half, and full time step.

Next, we express the algorithm that incorporates the leapfrog method in the Bulirsch-Stoer extrapolation scheme over a step $H$, which is split into $n$ substeps of size $h=H / n$, as follows:

$$
\begin{aligned}
\mathbf{q}_{1 / 2} & =\mathbf{q}_{0}+\frac{h}{2} \frac{\dot{\mathbf{q}}\left(\mathbf{W}_{0}^{\mathbf{q}}, \boldsymbol{\rho}_{0}, W_{0}^{t}\right)}{\Omega\left(\mathbf{W}_{0}^{\mathbf{q}}\right)}, \\
\mathbf{W}_{1 / 2}^{\rho} & =\mathbf{W}_{0}^{\rho}+\frac{h}{2} \frac{\dot{\boldsymbol{\rho}}\left(\mathbf{W}_{0}^{\mathbf{q}}, \boldsymbol{\rho}_{0}, W_{0}^{t}\right)}{\Omega\left(\mathbf{W}_{0}^{\mathbf{q}}\right)}, \\
t_{1 / 2} & =t_{0}+\frac{h}{2} \frac{1}{\Omega\left(\mathbf{W}_{0}^{\mathbf{q}}\right)}, \\
\mathbf{W}_{1}^{\mathbf{q}} & =\mathbf{W}_{0}^{\mathbf{q}}+h \frac{\dot{\mathbf{q}}\left(\mathbf{q}_{1 / 2}, \mathbf{W}_{1 / 2}^{\rho}, t_{1 / 2}\right)}{\Omega\left(\mathbf{q}_{1 / 2}\right)} \\
\rho_{1} & =\rho_{0}+h \frac{\dot{\rho}\left(\mathbf{q}_{1 / 2}, \mathbf{W}_{1 / 2}^{\rho}, t_{1 / 2}\right)}{\Omega\left(\mathbf{q}_{1 / 2}\right)},
\end{aligned}
$$

$$
\begin{gathered}
W_{1}^{t}=W_{0}^{t}+h \frac{1}{\Omega\left(\mathbf{q}_{1 / 2}\right)}, \\
\vdots \\
\mathbf{q}_{m-1 / 2}=\mathbf{q}_{m-3 / 2}+h \frac{\dot{\mathbf{q}}\left(\mathbf{W}_{\mathrm{m}-1}^{\mathbf{q}}, \boldsymbol{\rho}_{\mathrm{m}-1}, W_{m-1}^{t}\right)}{\Omega\left(\mathbf{W}_{\mathrm{m}-1}^{\mathbf{q}}\right)}, \\
\mathbf{W}_{\mathrm{m}-1 / 2}^{\rho}=\mathbf{W}_{\mathrm{m}-3 / 2}^{\rho}+h \frac{\dot{\rho}\left(\mathbf{W}_{\mathrm{m}-1}^{\mathbf{q}}, \boldsymbol{\rho}_{\mathrm{m}-1}, W_{m-1}^{t}\right)}{\Omega\left(\mathbf{W}_{\mathrm{m}-1}^{\mathbf{q}}\right)}, \\
t_{m-1 / 2}=t_{m-3 / 2}+h \frac{1}{\Omega\left(\mathbf{W}_{\mathrm{m}-1}^{\mathbf{q}}\right)},
\end{gathered}
$$$$
\mathbf{W}_{\mathrm{m}}^{\mathbf{q}}=\mathbf{W}_{\mathrm{m}-1}^{\mathbf{q}}+h \frac{\dot{\mathbf{q}}\left(\mathbf{q}_{\mathrm{m}-1 / 2}, \mathbf{W}_{\mathrm{m}-1 / 2}^{\rho}, t_{m-1 / 2}\right)}{\Omega\left(\mathbf{q}_{\mathrm{m}-1 / 2}\right)},
$$$$
\rho_{\mathrm{m}}=\rho_{\mathrm{m}-1}+h \frac{\dot{\rho}\left(\mathbf{q}_{\mathrm{m}-1 / 2}, \mathbf{W}_{\mathrm{m}-1 / 2}^{\rho}, t_{m-1 / 2}\right)}{\Omega\left(\mathbf{q}_{\mathrm{m}-1 / 2}\right)},
$$$$
W_{m}^{t}=W_{m-1}^{t}+h \frac{1}{\Omega\left(\mathbf{q}_{\mathrm{m}-1 / 2}\right)},
$$$$
\mathbf{q}_{\mathrm{n}}=\mathbf{q}_{\mathrm{n}-1 / 2}+\frac{h}{2} \frac{\dot{\mathbf{q}}\left(\mathbf{W}_{\mathrm{n}}^{\mathbf{q}}, \boldsymbol{\rho}_{\mathrm{n}}, W_{n}^{t}\right)}{\Omega\left(\mathbf{W}_{\mathrm{n}}^{\mathbf{q}}\right)},
$$$$
\mathbf{W}_{\mathrm{n}}^{\rho}=\mathbf{W}_{\mathrm{n}-1 / 2}^{\rho}+\frac{h}{2} \frac{\dot{\rho}\left(\mathbf{W}_{\mathrm{n}}^{\mathbf{q}}, \rho_{\mathrm{n}}, W_{\mathrm{n}}^{t}\right)}{\Omega\left(\mathbf{W}_{\mathrm{n}}^{\mathbf{q}}\right)},
$$$$
t_{n}=t_{n-1 / 2}+h \frac{1}{\Omega\left(\mathbf{W}_{\mathrm{n}}^{\mathbf{q}}\right)} \text {, }
$$

where $m=2, \ldots, n$.
[1] H. R. Reiss, Limits on Tunneling Theories of Strong-Field Ionization, Phys. Rev. Lett. 101, 043002 (2008).

[2] H. R. Reiss, The tunnelling model of laser-induced ionization and its failure at low frequencies, J. Phys. B 47, 204006 (2014).

[3] C. T. L. Smeenk, L. Arissian, B. Zhou, A. Mysyrowicz, D. M. Villeneuve, A. Staudte, and P. B. Corkum, Partitioning of the Linear Photon Momentum in Multiphoton Ionization, Phys. Rev. Lett. 106, 193002 (2011).

[4] A. Ludwig, J. Maurer, B. W. Mayer, C. R. Phillips, L. Gallmann, and U. Keller, Breakdown of the Dipole Approximation in Strong-Field Ionization, Phys. Rev. Lett. 113, 243001 (2014).

[5] B. Wolter, M. G. Pullen, M. Baudisch, M. Sclafani, M. Hemmer, A. Senftleben, C. D. Schröter, J. Ullrich, R. Moshammer, and J. Biegert, Strong-Field Physics with Mid-IR Fields, Phys. Rev. X 5, 021034 (2015).

[6] J.-L. Bégin, M. Alsaawy, and R. Bhardwaj, Chiral discrimination by recollision enhanced femtosecond laser mass spectrometry, Sci. Rep. 10, 14074 (2020).

[7] S. Chelkowski and A. D. Bandrauk, Photon momentum transfer in photoionisation: Unexpected breakdown of the dipole approximation, Mol. Phys. 115, 1971 (2017).
[8] M.-X. Wang, S.-G. Chen, H. Liang, and L.-Y. Peng, Review on non-dipole effects in ionization and harmonic generation of atoms and molecules, Chin. Phys. B 29, 013302 (2020).

[9] S. Palaniyappan, A. DiChiara, E. Chowdhury, A. Falkowski, G. Ongadi, E. L. Huskins, and B. C. Walker, Ultrastrong field ionization of $\mathrm{Ne}^{n+}(n \leqslant 8)$ : Rescattering and the Role of the Magnetic Field, Phys. Rev. Lett. 94, 243003 (2005).

[10] M. Y. Emelin and M. Y. Ryabikin, Atomic photoionization and dynamical stabilization with subrelativistically intense highfrequency light: Magnetic-field effects revisited, Phys. Rev. A 89, 013418 (2014).

[11] C. C. Chirilă, N. J. Kylstra, R. M. Potvliege, and C. J. Joachain, Nondipole effects in photon emission by laser-driven ions, Phys. Rev. A 66, 063411 (2002).

[12] M. W. Walser, C. H. Keitel, A. Scrinzi, and T. Brabec, High Harmonic Generation Beyond the Electric Dipole Approximation, Phys. Rev. Lett. 85, 5082 (2000).

[13] C. H. Keitel and P. L. Knight, Monte Carlo classical simulations of ionization and harmonic generation in the relativistic domain, Phys. Rev. A 51, 1420 (1995).

[14] S. Chelkowski, A. D. Bandrauk, and P. B. Corkum, Photon Momentum Sharing Between an Electron and an Ion in 
Photoionization: From One-Photon (Photoelectric Effect) to Multiphoton Absorption, Phys. Rev. Lett. 113, 263005 (2014).

[15] S. Chelkowski, A. D. Bandrauk, and P. B. Corkum, Photonmomentum transfer in multiphoton ionization and in timeresolved holography with photoelectrons, Phys. Rev. A 92, 051401(R) (2015).

[16] A. S. Titi and G. W. F. Drake, Quantum theory of longitudinal momentum transfer in above-threshold ionization, Phys. Rev. A 85, 041404(R) (2012).

[17] I. A. Ivanov, Relativistic calculation of the electron-momentum shift in tunneling ionization, Phys. Rev. A 91, 043410 (2015).

[18] S.-G. Chen, W.-C. Jiang, S. Grundmann, F. Trinter, M. S. Schöffler, T. Jahnke, R. Dörner, H. Liang, M.-X. Wang, L.-Y. Peng, and Q. Gong, Photon Momentum Transfer in SinglePhoton Double Ionization of Helium, Phys. Rev. Lett. 124, 043201 (2020).

[19] S. Chelkowski and A. D. Bandrauk, Photon-momentum transfer in molecular photoionization, Phys. Rev. A 97, 053401 (2018).

[20] P. B. Corkum, Plasma Perspective on Strong Field Multiphoton Ionization, Phys. Rev. Lett. 71, 1994 (1993).

[21] K. T. Taylor, J. S. Parker, D. Dundas, and K. J. Meharg, Theory of laser-driven double-ionization of atoms at Ti:sapphire laser wavelengths, J. Mod. Opt. 54, 1959 (2007).

[22] A. Becker, R. Dörner, and R. Moshammer, Multiple fragmentation of atoms in femtosecond laser pulses, J. Phys. B 38, S753 (2005).

[23] A. Emmanouilidou and T. Meltzer, Recollision as a probe of magnetic-field effects in nonsequential double ionization, Phys. Rev. A 95, 033405 (2017).

[24] A. Emmanouilidou, T. Meltzer, and P. B. Corkum, Non-dipole recollision-gated double ionization and observable effects, J. Phys. B 50, 225602 (2017).

[25] F. Sun, X. Chen, W. Zhang, J. Qiang, H. Li, P. Lu, X. Gong, Q. Ji, K. Lin, H. Li, J. Tong, F. Chen, C. Ruiz, J. Wu, and F. He, Longitudinal photon-momentum transfer in strong-field double ionization of argon atoms, Phys. Rev. A 101, 021402(R) (2020).

[26] Y. Mi, P. Peng, N. Camus, X. Sun, P. Fross, D. Martinez, Z. Dube, P. B. Corkum, D. M. Villeneuve, A. Staudte, R. Moshammer, and T. Pfeifer, Clocking Enhanced Ionization of Hydrogen Molecules with Rotational Wave Packets, Phys. Rev. Lett. 125, 173201 (2020).

[27] A. Emmanouilidou and A. Staudte, Intensity dependence of strong-field double-ionization mechanisms: From field-assisted recollision ionization to recollision-assisted field ionization, Phys. Rev. A 80, 053415 (2009).

[28] A. Chen, M. Kübel, B. Bergues, M. F. Kling, and A. Emmanouilidou, Non-sequential double ionization with near-single cycle laser pulses, Sci. Rep. 7, 7488 (2017).

[29] H. Price, C. Lazarou, and A. Emmanouilidou, Toolkit for semiclassical computations for strongly driven molecules: Frustrated ionization of $\mathrm{H}_{2}$ driven by elliptical laser fields, Phys. Rev. A 90, 053419 (2014).

[30] A. Emmanouilidou, C. Lazarou, A. Staudte, and U. Eichmann, Routes to formation of highly excited neutral atoms in the breakup of strongly driven $\mathrm{H}_{2}$, Phys. Rev. A 85, 011402(R) (2012).
[31] A. Chen, H. Price, A. Staudte, and A. Emmanouilidou, Frustrated double ionization in two-electron triatomic molecules, Phys. Rev. A 94, 043408 (2016).

[32] B. Manschwetus, T. Nubbemeyer, K. Gorling, G. Steinmeyer, U. Eichmann, H. Rottke, and W. Sandner, Strong Laser Field Fragmentation of $\mathrm{H}_{2}$ : Coulomb Explosion Without Double Ionization, Phys. Rev. Lett. 102, 113002 (2009).

[33] J. McKenna, A. M. Sayler, B. Gaire, N. G. Kling, B. D. Esry, K. D. Carnes, and I. Ben-Itzhak, Frustrated tunnelling ionization during strong-field fragmentation of $\mathrm{D}_{3}^{+}$, New J. Phys. 14, 103029 (2012).

[34] P. Pihajoki, Explicit methods in extended phase space for inseparable Hamiltonian problems, Celestial Mech. Dyn. Astron. 121, 211 (2015).

[35] L. Liu, X. Wu, G. Huang, and F. Liu, Higher order explicit symmetric integrators for inseparable forms of coordinates and momenta, Mon. Not. R. Astron. Soc. 459, 1968 (2016).

[36] R. Murray, M. Spanner, S. Patchkovskii, and M. Y. Ivanov, Tunnel Ionization of Molecules and Orbital Imaging, Phys. Rev. Lett. 106, 173001 (2011).

[37] N. B. Delone and V. P. Krainov, Energy and angular electron spectra for the tunnel ionization of atoms by strong low-frequency radiation, J. Opt. Soc. Am. B 8, 1207 (1991).

[38] N. B. Delone and V. P. Krainov, Tunneling and barriersuppression ionization of atoms and ions in a laser radiation field, Phys. Usp. 41, 469 (1998).

[39] L. Fechner, N. Camus, J. Ullrich, T. Pfeifer, and R. Moshammer, Strong-Field Tunneling from a Coherent Superposition of Electronic States, Phys. Rev. Lett. 112, 213001 (2014).

[40] H. Niikura, F. Légaré, R. Hasbani, A. D. Bandrauk, M. Y. Ivanov, D. M. Villeneuve, and P. B. Corkum, Sub-lasercycle electron pulses for probing molecular dynamics, Nature (London) 417, 917 (2002).

[41] T. Zuo and A. D. Bandrauk, Charge-resonance-enhanced ionization of diatomic molecular ions by intense lasers, Phys. Rev. A 52, R2511(R) (1995).

[42] T. Seideman, M. Y. Ivanov, and P. B. Corkum, Role of Electron Localization in Intense-Field Molecular Ionization, Phys. Rev. Lett. 75, 2819 (1995).

[43] D. M. Villeneuve, M. Y. Ivanov, and P. B. Corkum, Enhanced ionization of diatomic molecules in strong laser fields: A classical model, Phys. Rev. A 54, 736 (1996).

[44] E. Dehghanian, A. D. Bandrauk, and G. Lagmago Kamta, Enhanced ionization of the $\mathrm{H}_{2}$ molecule driven by intense ultrashort laser pulses, Phys. Rev. A 81, 061403(R) (2010).

[45] E. Yakaboylu, M. Klaiber, H. Bauke, K. Z. Hatsagortsyan, and C. H. Keitel, Relativistic features and time delay of laser-induced tunnel ionization, Phys. Rev. A 88, 063421 (2013).

[46] D. C. Heggie, A global regularisation of the gravitational $n$ body problem, Celestial Mech. 10, 217 (1974).

[47] W. H. Press, S. A. Teukolsky, W. T. Vetterling, and B. P. Flannery, Numerical Recipes: The Art of Scientific Computing, 3rd ed. (Cambridge University Press, Cambridge, UK, 2007).

[48] R. Bulirsch and J. Stoer, Numerical treatment of ordinary differential equations by extrapolation methods, Numer. Math. 8, 1 (1966). 
[49] D. H. Kobe and K-H. Yang, Energy of a classical charged particle in an external electromagnetic field, Eur. J. Phys. 8, 236 (1987).

[50] J. G. Leopold and I. C. Percival, Ionisation of highly excited atoms by electric fields. III. Microwave ionisation and excitation, J. Phys. B 12, 709 (1979).
[51] N. Camus, B. Fischer, M. Kremer, V. Sharma, A. Rudenko, B. Bergues, M. Kübel, N. G. Johnson, M. F. Kling, T. Pfeifer, J. Ullrich, and R. Moshammer, Attosecond Correlated Dynamics of Two Electrons Passing Through a Transition State, Phys. Rev. Lett. 108, 073003 (2012). 